BULL. AUSTRAL. MATH. SOC.

VOL. 9 (1973), 10்9-120.

\title{
Uniqueness of invariant means on certain introverted spaces
}

\section{Marvin W. Grossman}

Let $S$ be a topological semigroup with separately continuous multiplication and $H$ a uniformly closed invariant subspace of LUC(S) (the space of left uniformly continuous bounded functions on $S$ ) that contains the constants. It is shown that if $H$ is left introverted and $H$ admits a tight two-sided invariant mean $m$, then for each $h \in H, m(h)$ is the unique constant function in the norm closed convex hull of the left orbit of $h$; consequently, $H$ has a unique left invariant mean. (In fact, it is enough for $H$ to admit a tight right invariant mean and a left invariant mean.) For certain $S$, a similar result is obtained when $H$ is a left compact-open introverted subspace of LCC $(S)$ (the space of left compact-open continuous functions on $S$ ).

\section{Introduction}

The following theorem, which is a generalization of the uniqueness of Haar measure on a compact topological group, is apparently well-known (see for example, [3, p. 10] for the case when $S$ has an identity and $H=W(S)$ ).

THEOREM. Let $S$ be a topological semigroup with separately continuous multiplication and $H$ a zoniformly closed invariant subspace of $W(S)$ (the space of weakly almost periodic functions on $S$ ). If $H$ admits a two-sided invariant mean $m$, then for each $h \in H, m(h)$ is the

Received 20 March 1973. This research was supported in jart by a Temple University research grant. 
unique constant fronction in the norm closed convex hull of the right orbit of $h$ and the unique constant function in the norm closed convex hull of the left orbit of $h$; in particular, $H$ has a unique two-sided, left or might invariant mean.

A quick proof, part of the folklore, of this theorem is as follows. Let $h \in H$ and let $K_{h}$ be the norm (weakly) closed convex hull of the right orbit $R(h)$ of $h$. Then $K_{h}$ is weakly compact (Krein-Smulian) and $m(h)$ is in the pointwise closed convex hull of $R(h)$ (see for example, [9, Theorem 1]). Since the pointwise topology is weaker than the weak topology, the pointwise and weak topologies coincide on $K_{h}$ and therefore $m(h)$ is in the norm closed convex hull of $R(h)$. The argument for $L(h)$ is similar since $L(h)$ is necessarily weakly relatively compact (for example, [3]). The uniqueness assertions are then obvious (see the proof of Theorem 1 below).

In this paper we obtain two theorems of the above type, one for tight invariant means on introverted subspaces of $\operatorname{LUC}(S)$ and one for compactopen continuous invariant means on compact-open introverted subspaces of $\operatorname{LCC}(S)$ (the compact-open analogue of $\operatorname{LUC}(S)$ ). The motivation for the proofs of these theorems was an alternative argument of the author (in the spirit of [3, pp. 10, 11]) for the theorem stated above under the additional assumption that $H$ is introverted. The basic tool employed was a form of Day's fixed point theorem due to Argabright (see [17], [2]). For the proofs of our main theorems we employ two fixed point results of the latter type whose proofs are implicitly contained in the treatment of Section 4 in [10].

\section{Definitions and notations}

Throughout this paper a topological semigroup is a semigroup equipped with a Hausdorff topology for which multiplication is separately continuous. If $S$ is a topological semigroup, then $\mathrm{R}^{S}(m(S))$ denotes the space of all (bounded) real-valued functions on $S$ and $C(S)(B C(S))$ the space of all (bounded) continuous real-valued functions on $S$. If $f \in \mathrm{R}^{S}$ and $s \in S$, then $f_{s} ; f^{S}$ denote the functions defined on $S$ by 
$f_{s}(a)=f(s a)$ and $f^{s}(a)=f(a s)$ for all $a \in S$. If. $f \in R^{S}$, then $R(f)$ denotes the right orbit $\left\{f^{s}: s \in S\right\}$ of $f$ and $L(f)$ the left orbit $\left\{f_{s}: s \in S\right\}$ of $f$. A linear subspace $H$ of $\mathbb{R}^{S}$ is invariant if $h_{s} \in H$ and $h^{s} \in H$ for all $h \in H$ and all $s \in S$. An invariant subspace $H$ of $m(S)$ is left (right) introverted if for every $\psi \in H^{*}$ (the Banach dual of $H$ ) and every $h \in H, \psi\left(h_{s}\right)\left(\psi\left(h^{s}\right)\right)$ considered as a function of $s$ lies in $H$. Similarly, an invariant subspace $H$ of $\mathrm{R}^{S}$ is left (right) compact-open introverted if the latter condition holds where $H^{*}$ is the dual space of $H$ with. $H$ equipped with the compact-open topology (the topology of uniform convergence on compacta).

The space of left uniformly contintous functions on $S$, denoted $\operatorname{LUC}(S)$, is the set of $f$ in $B C(S)$ such that the map $s \rightarrow f_{s}$ is continuous where $B C(S)$ has the sup norm. The space of left compact-open continuous functions on $S$, denoted $\operatorname{LCC}(S)$, is the set of $f$ in $C(S)$ such that the map $s \rightarrow f_{s}$ is continuous where $C(S)$ has the compact-open topolosy. In a similar manner, one defines the space $\operatorname{RUC}(S)$ of right coniformly continuous functions on $S$ and the space $\operatorname{RCC}(S)$ of right compact-open continuous functions on $S$.

Let $H$ be an invariant subspace of $\mathrm{R}^{S}$ that contains the constant functions. A linear functional $m$ on $H$ is said to be a mean on $H$ if $m(h) \geq 0$ for all $h \in H$ with $h \geq 0$ and $m(1)=1 . m$ is a left (right) invariant mean on $H$ if $m$ is a mean on $H$ and $m\left(h_{\mathcal{S}}\right)=m(h)$ $\left(m\left(h^{s}\right)=m(h)\right)$ for all $h \in H$ and all $s \in S . m$ is two-sided invariant if $m$ is both left and right invariant.

If $K$ is a convex subset of a real locally convex Hausdorff space $E$, then $E(K)$ denotes the set of restrictions of the real-valued continuous affine functions on $E$ to $K$; that is, $E(K)=E^{\star} \mid K+\mathrm{R}$ and $A(K)$ denotes the space of all real-valued continuous affine functions on $K$. $B E(K)$ and $B A(K)$ denote respectively the bounded functions in $E(K)$ and $A(K)$. If $S$ is a semigroup and $K$ is a convex subset of $E$ with 
the relative topology, then an (restricted) affine action of $S$ on $K$ denoted $(S, K)$, is a map from $S \times K$ into $K,(s, x) \rightarrow s \cdot x$, such that

(1) $s_{1} \cdot\left(s_{2} \cdot x\right)=\left(s_{1} s_{2}\right) \cdot x$ for all $s_{1}, s_{2} \in S$ and all $x \in K$;

(2) for each $s \in S$, the map $x \rightarrow s \cdot x$ is a (restriction of a) continuous affine map of $K(E)$ into $K(E)$.

If $(S, K)$ is an affine action of $S$ on $K$ and $x \in K$, then

$T x: A(K) \rightarrow \mathrm{R}^{S}$ is the map defined by $T x f(s)=f(s \cdot x)$ for all $f \in A(K)$ and all $s \in S$.

\section{Two fixed point results}

The two lemmas below were stated in [10]. Lemma 1 is a slight generalization of a result of Khurana [13, Theorem 2.1]. We include a proof for the sake of completeness (see the proof of Theorem 2.2 in [13]).

DEFINITION. Let $X$ be a Hausdorff topological space and $H$ a linear space of bounded continuous real-valued.functions on $X$ that contains the constant functions. A mean $\mu$ on $H$ ( $\mu$ is linear, $\mu(h) \geq 0$ for $h \geq 0$ and $\mu(1)=1$ ) is said to be tight if for each uniformly bounded net $\left\{h_{\alpha}\right\}$ in $H$ with $h_{\alpha} \rightarrow 0$ uniformly on compact subsets of $X$, we have $\mu\left(h_{\alpha}\right) \rightarrow 0$. A mean $\mu$ on $H$ is $\sigma$-additive if for each sequence $\left\{h_{n}\right\} \subset H$ with $h_{n} \downarrow 0$, we have $\mu\left(h_{n}\right) \rightarrow 0$.

LEMMA 1. Let $K$ be a complete bounded convex subset of a locally convex Hausdorff space $E$. If $\mu$ is a tight mean on $E(K)$ (respectively $B A(K)$ ), then there exists a zonique $x_{0}$ in $K$ such that $\mu(f)=f\left(x_{0}\right)$ for alz $f$ in $E(K)$ (respectively $B A(K)$ ).

Proof. Let $\tilde{E}$ be the completion of $E$ and equip $\tilde{E}^{*}$ with the weak topology $\sigma\left(\tilde{E}^{*}, \tilde{E}\right)$. Define the linear functional $\hat{\mu}: \tilde{E}^{*} \rightarrow \mathrm{R}$ by $\hat{\mu}(g)=\mu(g \mid K)$ for all $g \in \tilde{E}^{*}$. We need only show that $\hat{\beta}$ is $\sigma\left(E^{*}, \tilde{E}\right)-$ continuous. For then $\hat{\mu}$ must be the evaluation functional at some $x_{0} \in \tilde{E}$ and since $K$ is closed convex in $\tilde{E}$ we must have $x_{0} \in K \cdot x_{0}$ is unique since $E(K)$ separates points. (If $\mu$ is a tight mean on $B A(K)$, then $\mu$ is the restriction of a finitely additive positive measure of total mass 
one on $K$ (Hahn-Banich) and therefore by [14, Theorem 1] $\mu(f)=f\left(x_{0}\right)$ for $f$ in $B A(K)$ as well.)

In order to show that $\hat{\rho}$ is $\sigma\left(\tilde{E}^{*}, \tilde{E}\right)$-continuous it suffices by $[12$, p. 156] to verify that $\hat{\mu}$ is continuous on every equicontinuous subset $G$ of $\tilde{E}^{*}$. Let $\left\{g_{\alpha}\right\}$ be a net in $G$ and $g \in G$ such that $g_{\alpha} \rightarrow g$ pointwise on $\tilde{E}$. Since $K$ is bounded and $G$ is equicontinuous, $\left\{g_{\alpha}\right\}$ is uniformly bounded on $K$. Since $G$ is equicontinuous and $g_{\alpha} \rightarrow g$ pointwise on $\tilde{E}, g_{\alpha} \rightarrow g$ uniformly on totally bounded sets $[12, p .76]$. Since $\mu$ is tight, $\mu\left(g_{\alpha} \mid K\right) \rightarrow \mu(g \mid K)$.

A slight modification of the proof of Lemma 1 yields

LEMMA 2. Let $K$ be a complete convex subset of a locally convex Hausdorff space $E$. If $\mu$ is a mean on $E(K)$ which is continuous when $E(K)$ has the compact-open topology, then there exists a unique $x_{0}$ in $K$ such that $\mu(f)=f\left(x_{0}\right)$ for all $f$ in $E(K)$.

The following definitions are adapted from Mitchell [17] and Argabright [2].

Let $S$ be a topological semigroup and $H$ a subset of $C(S)$. Let $(S, K)$ be a (restricted) affine action of $S$ on $K$ where $K$ is a convex subset with relative topology of a locally convex space $E$. The action $(S, K)$ is an E-representation of $S, H$ on $K$ by (restricted) continuous affine maps if there is an $x$ in $K$ such that the map $s+s \cdot x$ is continuous and $T x(A(K)) \subset H \quad(T x(E(K)) \subset H)$. The action $(S, K)$ is a bounded E-representation of $S, H$ on $K$ by (restricted) continuous affine maps if there is an $x$ in $K$ such that $s+s \cdot x$ is continuous and $T x(B A(K)) \subset H \quad(T x(B E(K)) \subset H)$. Let $K$ be a class of convex subsets of locally convex Hausdorff spaces. The pair $S, H$ has the common fixed point property on the sets in $K$ with respect to E-representations by (restricted) affine mops. if for each $K$ in $K$ and $E$-representation $(S, K)$ of $S, H$ by (restricted) continuous affine maps, $K$ has a common fixed point for the action of $S$. The common fixed point property with respect to bounded $E$-representations is defined similarly.

REMARK. Note that if $K$ is compact and $x \in K$ with $T x(E(K)) \subset H$, 
then necessarily $s \rightarrow s \cdot x$ is continuous. Consequently, the above definition of an $E$-representation is consistent with that given by Argabright in [2].

The following two propositions are implicit in the proofs of Proposition 4.7 and "(1) implies (2)" of Proposition 4.13 in [10]. The technique of the proof appears throughout the literature (see, for example, [17], [2]). We include the proof since it is so short.

PROPOSITION 1. Let $S$ be a topological semigroup and $H$ an invariant subspace of $B C(S)$ that contains the constant functions. If $H$ admits a tight left invariant mean, then $S, H$ has the common fixed point property on complete bounded convex sets with respect to E-representations by restricted affine maps and on complete bounded convex sets with respect to bounded E-representation by affine maps.

Proof. Let $(S, K)$ be an $E$-representation of $S, H$ by restricted continuous affine maps on the complete bounded convex set $K$. Let $x \in K$ be such that $s \rightarrow s^{*} x$ is continuous and $T x(E(K)) \subset H$. If $T x^{*}: H^{*} \rightarrow E(K)^{*}$ is the adjoint of $T x$ and $m$ is a tight left invariant mean on $H$, then $T x^{*} m$ is a tight mean on $E(K)$ which is invariant under the action of $S$ on $K$. Consequently, by Lemma 1 , there is an $x_{0}$ in $K$ such that $f\left(s \cdot x_{0}\right)=f\left(x_{0}\right)$ for all $f \in E(K)$ and all $s \in S$. Since $E(K)$ separates points, $s \cdot x_{0}=x_{0}$ for all $s \in S$. The argument is similar for bounded $E$-representations by continuous affine maps.

Applying in the above proof Lemma 2 in place of Lemma 1, we obtain

PROPOSITION 2. Let $S$ be a topological semigroup and $H$ an invariant subspace of $C(S)$ that contains the constant functions. If $H$ admits a compact-open continuous left invariant mean, then $S, H$ has the common fixed point property on complete convex sets with respect to E-representations by restricted affine maps.

REMARK. We note that if in Proposition 2, $S$ is realcompact and for every $f \in C(S)$ with $f \geq 0$ there exists $h \in H$ such that $f \leq h$, then every mean on $H$ is compact-open continuous. For every mean on $H$ has an extension to a mean on $C(S)$ [18, p. 82] and every mean on $C(S)$ is necessarily compact-open continuous [6, Theorem 5.3]. 


\section{The main theorems}

We now apply Propositions 1 and 2 to obtain our main results. The proof of the following theorem is an adaptation of the proof of Lemma 5.1 in [4] to our present setting ( $c f$. also [3, pp. 10, 11]).

THEOREM 1. Let $S$ be a topological semigroup and $H$ a uniformly closed invariant subspace of $\operatorname{LUC}(S)$ (respectively $\operatorname{RUC}(S)$ ) that contains the constant functions and is left (respectively right) introverted. If $H$ admits a tight two-sided invariant mean $m$, then for each $h \in H, m(h)$ is the unique constant fronction in the norm closed convex hull of $L(h)$ (respectively $R(h)$ ); in particular, $H$ has a unique left (respectively right) invariant mean.

Proof. We assume $H$ is right introverted and $H \subset \operatorname{RUC}(S)$. The proof for $H$ left introverted with $H \subset \operatorname{LUC}(S)$ follows then by considering the multiplication $s_{1} \circ s_{2}=s_{2} s_{1}$ on $S$. If for every $h$ in $H$ the norm closed convex hull $K_{h}$ of $R(h)$ in $H$ contains the constant function $m(h)$, then necessarily $H$ has a unique right invariant mean. For if $\nu$ is a right invariant mean on $H$, then by linearity and continuity of $\nu$ we must have $\nu(m(h))=v(h)$; that is, $m(h)=v(h)$. We show, in fact, that $m(h)$ is the unique function $g$ in $K_{h}$ such that $g=g^{s}$ for all $s \in S$.

Fix $h \in H$ and let $\left(S, K_{h}\right)$ denote the left action of $S$ on $K_{h}$ defined by $s \cdot f=f^{s}$ for all $s \in S$ and all $f \in K_{h}$. Then $\left(s, K_{h}\right)$ is a restricted affine action of $S$ on $k_{h}$ (for fixed $s \in S$, the map $f \rightarrow f^{S}$ on $H$ is linear and norm continuous). Now let $f \in K_{h}$ and consider the map If $: E\left(K_{h}\right) \rightarrow m(S)$. Since $f \in \operatorname{RUC}(S)$, the map $s \rightarrow f^{S}$ is continuous and since $H$ is right introverted with $1 \in H$, we have $\operatorname{Tf}\left(E\left(K_{h}\right)\right) \subset H$. Thus, $\left(S, K_{h}\right)$ is an E-representation of $S, H$ by restricted continuous affine maps on $K_{h}$ - a complete norm bounded convex subset of $H$. Since $H$ admits a tight left inveriant mean, the action $\left(S, K_{h}\right)$ has a common fixed point by Proposition 1 ; that is, there is a 
$g \in K_{h}$ such that $g=g^{s}$ for all $s \in S$.

Since $K_{h}$ is the norm closed convex hull of $R(h)$, for each $\varepsilon>0$, there exists a convex combination $\sum_{i=1}^{n} \lambda_{i} h^{s} i$ such that

$\left\|g-\sum_{i=1}^{n} \lambda_{i} h^{s} i\right\| \leq \varepsilon$. If we fix $x \in S$ and $\varepsilon>0$, then

$\left|g(x)-\sum_{i=1}^{n} \cdot \lambda_{i} h^{s}{ }^{i}(x s)\right| \leq \varepsilon$ for all $s \in S$; that is,

$\left|g(x)-\sum_{i=1}^{n} \lambda_{i}\left(h_{x}\right)^{s} i\right| \leq \varepsilon$. If we apply $m$ to the last inequality, we have $\left|g(x)-\sum_{i=1}^{n} \lambda_{i} m(h)\right| \leq \varepsilon$; that is, $|g(x)-m(h)| \leq \varepsilon$. Consequently, $m(h)=g(x)$ for all $x \in S$.

REMARKS. The above proof shows that in place of requiring $H \subset \operatorname{LUC}(S)$ (RUC $(S)$ ) it is sufficient to assume that the norm closed convex hull of $L(h) \quad(R(h))$ meets $\operatorname{LUC}(S)(\operatorname{RUC}(S))$ for every $h$ in $H$. Also, the theorem is valid if $H$ admits a tight right (left) invariant mean and a left (right) invariant mean where $m$ is taken to be a two-sided invariant mean (necessarily unique) on $H$. (Since $H$ is either left or right introverted, if $H$ has a left invariant mean and a right invariant mean, then $H$ has a two-sided invariant mean.) Under the additional assumption that $S$ has a right (left) identity it follows from the first part of the proof (see Theorem 2) that if $H$ admits a tight right (left) invariant mean, then the norm closed convex hull of $L(h)(R(h))$ contains a constant function for each $h$ in $H$.

It is of interest to note here a result of Granirer and Lau. In [9] it is shown that if $\operatorname{LUC}(S)$ has a left invariant mean $m$, then for each $h \in H, m(h)$ is in the compact-open closed convex hull of $R(h)$. Consequently, if LUC $(S)$ admits a compact-open continuous right invariant mean and a left invariant mean, then LUC(S) has a unique compact-open continuous right invariant mean.

COROLLARY. Let $S$ be a semigroup. If $m(S)$ admits a o-additive right (respectively left) invariont mean and a left (respectively right) 
invariant mean, then $m(S)$ has a voique left (respectively right) invariant mean.

Proof. If we equip $S$ with the discrete'topology, then $\operatorname{LUC}(S)=\operatorname{RUC}(S)=m(S)$. If $S$ is countable, then every $\sigma$-additive mean on $m(S)$ is tight (for example, [11, p. 40]) and therefore Theorem 1 applies (see the above remarks). For general $S$ a result of Granirer can be used. Namely, if $m(S)$ has a $\sigma$-additive left invariant mean, then $S$ contains a finite group which is a left ideal [7, Theorem 4.2]. Thus, every affine action of $S$ on a convex subset of a vector space has a common fixed point. The proof of Theorem 1 then remains valid for $S$.

REMARK. If in the corollary $S$ has left (right) cancellation, then $S$ is a finite group [8, Corollary 2.1].

THEOREM 2. Let $S$ be a topological semigroup with right (respectively left) identity $e$ such that $C(S)$ is complete in the compact-open topology (in particular, $S$ a completely regular $k$-space). Let $H$ be a compact-open closed invariant subspace of $\operatorname{LCC}(S)$ (respectively $\operatorname{RCC}(S)$ ) that contains the constant functions and is left (respectively right) compact-open introverted. If $H$ admits a compact-open continuous twosided invariant mean $m$, then for each $h \in H, m(h)$ is the unique constant function in the compact-open closed convex hull of $L(h)$ (respectively $R(h)$ ); in particular, $H$ has a unique compact-open continuous left (respectively right) invariant mean.

Proof. By applying Lemma 2 in place of Lemma 1, it follows exactly as in the proof of Theorem I that for each $h \in H$, there is a $g \in K_{h}$ (the compact-open closed convex hull of $R(h)$ ) with $g=g^{s}$ for all $s \in S$. Consequently, $g(e)=g(e \cdot s)=g(s)$ for all $s \in S$. It follows then $m(h)=g$ and $m$ is the unique compact-open continuous right invariant mean on $H$.

REMARKS. There exist spaces $S$ for which $C(S)$ is compact-open complete but $S$ is not a $k$-space [19, p. 363]. Again, it is enough to assume that the compact-open closed convex hull of $L(h)(R(h))$ meets $\operatorname{LCC}(S)(\operatorname{RCC}(S))$ for every $h$ in $H$. Of course, the argument shows that if $H$ admits a compact-open continuous right (left) invariant mesn, then the compact-open closed convex hull of $L(h)(R(h))$ contains a constant 
function for each $h$ in $H$.

COROLLARY. Let $S$ be a realcompact topological semigroup with jointly continuous product, right (respectively left) identity $e$ and $C(S)$ complete in the compact-open topology. Let $H$ be a compact-open closed invariant subspace of $C(S)$ such that $H$ contains the constants, is left (respectively right) compact-open introverted and satisfies: if $f \in C(S)$ with $f \geq 0$, then there exists $h \in H$ with $f \leq h$. If $H$ admits a two-sided invariant mean $m$, then for each $h \in H, m(h)$ is the unique constant function in the compact-open closed convex hull of $L(h)$ (respectively $R(h)$ ); in particular $H$ has a tonique left (respectively right) invariant mean.

Proof. Every mean on $H$ is necessarily compact-open continuous (see the remark in Section 2). Since multiplication on $S$ is jointly continuous, $\operatorname{LCC}(S)=\operatorname{RCC}(S)=C(S)$ (for example, [15, Lemna 4.2]).

REMARKS. In the above corollary $H$ can be taken to be $C(S)$ since $\operatorname{LCC}(S)=\operatorname{RCC}(S)=C(S)$ implies that $C(S)$ is both left and right compactopen introverted. For the case $H=C(S)$ Argabright in [1, Theorem 2.4] showed without an identity or completeness restriction that if $C(S)$ admits a two-sided invariant mean, then $C(S)$ has a unique left or right invariant mean.

We also note that the corollary is applicable to discrete semigrnups $S$ of non-measurable cardinal. For they are realcompact in the discrete topology [5, p. 163] and certainly $R^{S}$ is compact-open complete.

\section{References}

[1] L. Argabright, "Invariant means on topological semigroups", Pacific J. Math. 16 (1966), 193-203.

[2] L.N. Argabright, "Invariant means and fixed points; a sequel to Mitchell's paper", Troons. Arer. Math. Soc. 130 (1968), 127-130.

[3] R.B. Burckel, Weakly almost periodic functions on semigroups (Gordon and Breach, New York, London, Paris, 1970). 
[4] George Converse, I saac Namioka and R.R. Phelps, "Extreme invariant positive operators", Trans. Amer. Math. Soc. 137 (1969), $375-385$.

[5] Leonard Gillman and Meyer Jerison, Rings of continuous functions (Van. Nostrand, Princeton, New Jersey; Toronto; London; New York; 1960).

[6] G.G. Gould and M. Mahowald, "Measures on completely regular spaces", J. London Math. Soc. 37 (1962), 103-111.

[7] E. Granirer, "On amenable semigroups with a finite-dimensional set of invariant means I", Illinois J. Math. 7 (1963), 32-48.

[8] E. Granirer, "On amenable semigroups with a finite-dimensional set of invariant means II", IZZinois J. Math. 7 (1963), 49-58.

[9] E. Granirer and Anthony T. Lau, "Invariant means on locally compact groups", ILZinois J. Math. 15 (1971), 249-257.

[10] Marvin W. Grossman, "A categorical approach to invariant means and fixed point properties", Semigroup Forum 5 (1972), 14-44.

[11] Paul R. Halmos, Measure theory (Van Nostrand, Toronto, New York, London, 1950).

[12] J.L. Kelley, Isaac Namioka and W.F. Donoghue, Jr, Kenneth R. Lucas, B.J. Pettis, Ebbe Thue Poulsen, G. Baley Price, Wendy Robertson, W.R. Scott, Kennan T. Smith, Linear topological spaces (Van Nostrand, Princeton, New Jersey; Toronto; New York; London; 1963).

[13] Surjit Singh Khurana, "Measures and barycenters of measures on convex sets in locally convex spaces I", J. Math. Anal. Appl. 27 (1969), 103-115.

[14] Surjit Singh Khurana, "Measures and barycenters of measures on convex sets in locally convex spaces II", J. Math. Anal. Appl. 28 (1969), 222-229.

[15] Anthony To-ming Lau, "Action of topological semigroups, invariant means, and fixed points", Studia Math. 43 (1972), 139-156. 
[16] Theodore Mitchell, "Constant functions and left invariant means on semigroups", Trans. Amer. Math. Soc. 119 (1965), 244-261.

[17] Theodore Mitchell, "Function algebras, means, and fixed points", Trons. Amer. Math. Soc. 130 (1968), 117-126.

[18] Anthony L. Peressini, Ordered topological vector spaces (Harper and kow, New York, Evanston, London, 1967).

[19] Властиинл Птан [Vlastimil Pták], "О голных топологичесних линейных поостранствах" [On complete topological linear spaces], CehosZovack. Mat. Z. 3 (78) (1953), 301-364.

Department of Mathematics,

Temple University,

Philadelphia,

Pennsylvania,

USA. 